\title{
Design and Optimization of Rectangular Waveguide Filter based on Direct Coupled Resonators
}

\author{
Mehdi Damou, Keltouma Nouri, Mohammed Feham and Mohammed Chetioui
}

\begin{abstract}
The waveguide filter structure is treated by two softwares (HFSS (High Frequency Structure Simulator) and CST (Computer Simulation Technology)). Numerical example is given in this article to demonstrate, step by step, the application of the approach to the design of resonator, direct coupled waveguide and microstrip filters based on electromagnetic (EM) simulations. For this design procedure, the filter structure is simulated by successively adding one resonator at a time. To continue the work illustrates how to design a fourth order coupled resonator based rectangular waveguide circuit in the traditional way. With a large number of variables, such tuning work consumes a lot of time and the convergence of the final result is not guaranteed. A fourth order X-band bandpass filter with a center frequency of $11 \mathrm{GHz}$ and a fractional bandwidth FBW $=0,0273$ is designed using this procedure and presented here as an example. The simulated results by CST are presented and compared withthe results simulated by a high-frequency structure simulator. Good agreement between the simulated HFSSand simulated results by CST is observed.
\end{abstract}

Keywords-Coupling Matrix, Filter, Waveguide, technology, Electromagnetic, Bandpass Filter

\section{INTRODUCTION}

A microwave filter is a two-port network used to transmit and attenuate signals in specified frequency bands. Traditionally, the design methods for direct coupled filters have been applied to extract the dimensions for direct coupled filters. This design process usually involves the following four main steps: (i) identify the filter order and filter functions according to specification requirements; (ii) synthesis or optimise the coupling coefficients $(\mathrm{Mi}, \mathrm{j})$ and external quality factors $(\mathrm{Qe})$ that can realize the desired filter function; (iii) choose the filter type (waveguide, microstrip, etc), and obtain dimensions which can achieve desired specified $\mathrm{Qe}$ and $\mathrm{Mi}, \mathrm{j}$ from electromagnetic (EM) simulations on one resonator and two weakly coupled resonators; (iv) construct the filter in the simulator to get its initial responses [1, 2]. Coupled resonator circuits are of importance for design of RF/microwave filters with any type of resonator regardless its physical structure.

This work was co-financed by LTC

Damou Mehdi, Nouri Keltouma and Chetioui Mohammed are with Laboratory Technology of Communication, Faculty of Technology University Tahar Moulay of Saida, Algeria (bouazzamehdi@yahoo.fr; keltoum_nouri@ yahoo.fr).

Feham Mohammed Head of STIC Laboratory, Laboratory Systems and Technologies of Information and Communication University Tlemcen, Algeria (Feham_m@yahoo.fr).
The coupling matrix is used to represent the coupled resonator circuit. Each matrix entry value refers to a physical dimension of the circuit. The response of the circuit can also be calculated of the circuit. The response of the circuit can also be calculated by using the coupling matrix. Different methods are developed to generate the coupling matrix. This work presents design of the coupled resonator based filter by using the coupling matrix local optimization technique. The initial values of the matrix, which is used as the input of the optimizer, have a great effect on the convergence of the final result of the optimization. A proposed tuning technique, called Step Tune method, is also presented in this work. Instead of conventionally tuning the whole structure, we simulate and tune just part of the circuit by using the new method. As only limited number of physical dimensions is tuned each time, the final result is more reliable.

In this article, we present an EM-based design approach for determining the physical dimensions of a coupled waveguide filter with any type of topology. A design case of direct coupled waveguide filter will be discussed. Extractions for physical dimension by external quality factors and coupling coefficients are presented during the design procedures. Finally a proposed optimization technique, called Step Tune method has been developed, a design case of a direct-coupled waveguide filter will be presented in the end of this article.

\section{DESCRIPTION OF THE DESIGN}

The method, which is based on electromagnetic (EM) simulation, will be called the step tune method. Instead of traditionally altering all the parameters of the circuit in each optimizing iteration, the step-tune method simulates only one resonator of the device in the first step. When finishing tuning the first resonator, one more resonator is added and then the circuit is tuned or optimized again. More resonators are added successively to tuning at each step. For each step, a new coupling matrix is required for the tuning. As limited number of physical dimensions needs to optimize in each step, the optimizing process works more efficiently and generates more reliable solutions. The key point of this method is to calculate the $S$ parameters in each step and apply the responses as the objective ones for the physical optimizing. For this design method, the middle stage S-parameter responses are calculated from their corresponding coupling coefficients, and act as the objective responses for the tuning. To plot the desired responses at each stage, the inner coupling coefficient needs to be converted into external quality factor. For instance, at Step 1, 
$\mathcal{Q}_{e 2}$ should be calculated from $M_{12}$. After expressing both the external quality factor $\left(Q_{e}\right)$ and internal coupling coefficients $\left(M_{i, i+1}\right)$ using inverter value $\mathrm{K}[2]$, the relationship between $M_{i, i+1}$ and $\mathcal{Q}_{e}$ can be found as:

$$
M_{i, i+1}^{2} \cdot \mathcal{Q}_{e i}=\frac{1}{\frac{n \pi}{2}\left(\frac{\lambda g}{\lambda}\right)^{2} F B W}
$$

Where $\lambda_{g}$ is the guided wavelength of the resonant frequency and $\lambda$ is the free-space wavelength, $n$ is the number of halfwavelengths of the waveguide resonator cavity.

The coupling coefficients between external ports and inner resonators can be calculated by

$$
M_{i, p_{2}}=\sqrt{\frac{1}{Q_{e i, p_{2}}}}
$$

$i$ refer to the resonator number connecting to the output ports $\left(p_{2}\right)$. Substituting (1) into (2), we have

$$
M_{i, p 2}=\sqrt{\frac{n \pi}{2} F B W}\left(\frac{\lambda g}{\lambda}\right) M_{i, i+1}
$$

Where $M_{i, p 2}$ is the equivalent external coupling coefficient of the internal coupling iris.

\section{PHYSICAL OF COUPLING MATRIX}

After determining the normalized coupling matrix $[\mathrm{m}]$ for a coupled resonator topology, the actual coupling matrix $[M]$ of a coupled resonator device with given specification can be calculated by prototype de-normalization of the matrix $[\mathrm{m}]$ at a desired bandwidth, as follows [3]:

$$
\mathrm{M}_{\mathrm{i}, \mathrm{i}+1}=\mathrm{m}_{\mathrm{i}, \mathrm{i}+1} \cdot F B W \text { For } \mathrm{i}=1 \text { to } \mathrm{n}-1
$$

The actual external quality factor $Q_{e}$ is related to the normalized quality factor $q_{e}$ by [3]:

$$
\mathrm{Q}_{\mathrm{e}}=\frac{q_{e}}{F B W}
$$

The coupling elements $\mathbf{M}_{\mathrm{i}, \mathrm{i}+1}$ and external quality factors $\mathrm{Q}_{\mathrm{e} 1}, \mathrm{Q}_{\mathrm{en}}$ ) are related to the lumped element lowpass prototype elements $g_{0}, g_{1}, g_{2} \ldots g_{n+1}$ as follows [4]:

$$
\begin{aligned}
& Q_{e 1}=\frac{g_{0} g_{1}}{F B W}, \quad Q_{e n}=\frac{g_{n} g_{n+1}}{F B W} \\
& M_{i, j+1}=\frac{F B W}{\sqrt{g_{i} g_{i+1}}} \text { for } \mathrm{i}=1 \text { to } \mathrm{n}-1
\end{aligned}
$$

The circuit parameters can be related to the bandpass filter design parameters by the following equations:

Lumped LC elements of resonators [5]:

$$
\begin{aligned}
C_{0} & =\frac{Q_{e}}{\omega_{0} Z} \times 10^{12}(\mathrm{pF}) \\
L_{0} & =\frac{Z}{\omega_{0} Q_{e}} \times 10^{9}(\mathrm{nH})
\end{aligned}
$$

Lumped LC elements of resonators [5]:

$$
\begin{aligned}
& Z_{01}=Z_{45}=\mathrm{Z}(\mathrm{ohm}) \\
& Z_{12}=Z_{34}=\frac{Z}{M_{12} Q_{e}}, \quad Z_{23}=\frac{Z}{M_{23} Q_{e}}
\end{aligned}
$$

\section{APPliCATION OF THE DESIGN METHOD}

To demonstrate the technique for using the coupling matrix filter circuit model and an EM simulator to synthesize the physical dimensions of the filter, we consider the design of the proposed four-poles filter which. The Figure 1 illustrates the topology and the structure of this filter.

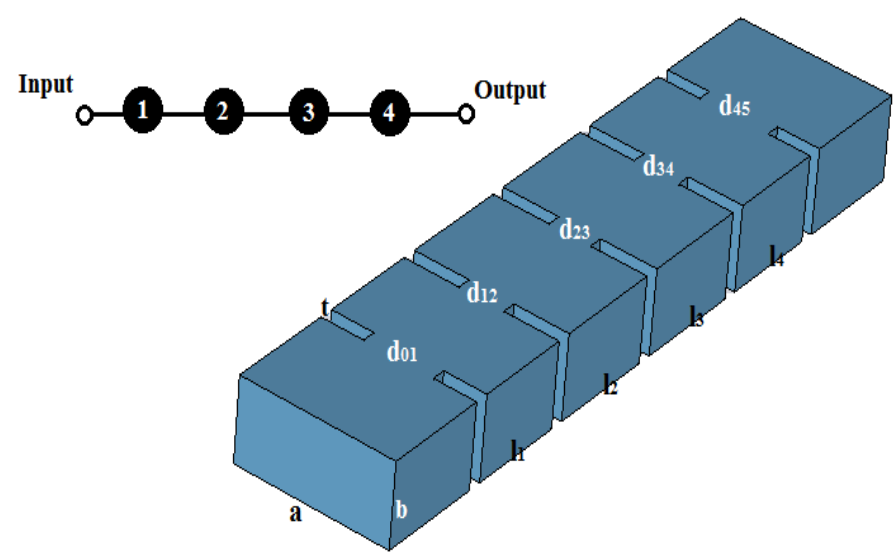

Fig. 1. Illustration of a four order X-band. These four resonators are operating at $\mathrm{TE}_{101}$ mode All the irises have the same thickness $\mathrm{t}$ of $2 \mathrm{~mm}, \mathrm{a}=22.86 \mathrm{~mm}$, $\mathrm{b}=10.16 \mathrm{~mm}$

The filter is a BPF at the center frequency $11 \mathrm{GHz}$, its pass band is $300 \mathrm{MHz}(2.7272 \%)$ and reflection loss of $20 \mathrm{~dB}$ at the passband, the out-of-band rejection is $45 \mathrm{~dB}$. These specifications can be achieved by Chebyshev response, since there is no transmission zeros in the response. With Chebyshev response, [6] for the desired bandwidth, the ripple in the pass band and the rejection, we found that the filter must be of the forth degree at least. The $n \times n$ coupling matrix cannot be used to derive the responses [8]. An $n+2$ coupling matrix $[M]_{n+2}$ is applied as

$$
\begin{gathered}
M=\left[\begin{array}{cccccc}
0 & -0.0282 & 0 & 0 & 0 & 0 \\
-0.0282 & 0 & 0.0249 & 0 & 0 & 0 \\
0 & 0.0249 & 0 & -0.0191 & 0 & 0 \\
0 & 0 & -0.0191 & 0 & 0.0249 & 0 \\
0 & 0 & 0 & 0.0249 & 0 & -0.0282 \\
0 & 0 & 0 & 0 & -0.0282 & 0
\end{array}\right] \\
\mathrm{Q}_{\mathrm{e} 1}=\mathrm{Q}_{\mathrm{e} 2}=\mathrm{Q}_{\mathrm{e}}=34.21
\end{gathered}
$$

\section{STRATEGIC OF THE STEPS}

The process of tuning the 4 th order filter can be divided into four steps. For convenience the selected steps can be represented by means of the pertinent diagram, where each circle represents a resonator and $M_{i, i+1}$ describe the coupling between resonators. The calculation of the physical dimensions for the fourth order pass-band filter shown in Figure 1comprises 
the following steps. In this way, the four stages selected in this design are represented schematically in figure 2 .

1. Calculate the approximate initial dimensions for all the resonators and irises using the equivalent circuit models based on the coupling matrix as described in $[1,6,7]$

2. Using the coupling matrix values calculated for the entire filter, obtain just the responses for the first resonator [see Fig. 2(a)]. Use the simulator (in our case, lwave wizard [8]) to evaluate resonator 1 together with its two adjacent irises, and optimize this simulated response toward the desired one from coupling matrix, by changing the resonator length $\left(l_{l}\right)$ and iris dimensions ( $d_{01}$ and $\left.d_{12}\right)$.

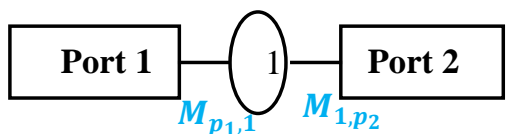

(a)

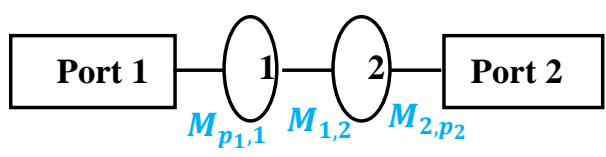

(b)

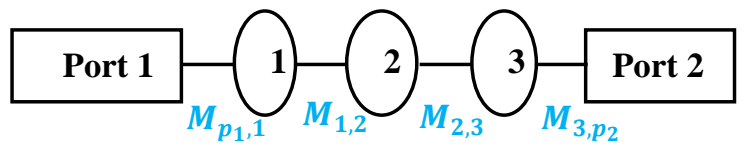

(c)

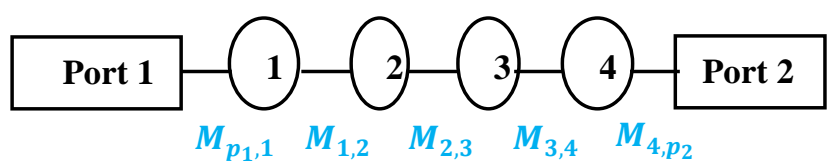

(d)

Fig. 2. Schematic representation of the steps of the filter design: a) first step, b) second step, c) third step, d) fourth step (final desired filter).

3. Use the EM simulator for both resonators 1 and 2 and their connecting irises [see Fig. 2(b)]. Adjust the length of resonator $2\left(l_{2}\right)$ and iris dimension $\left(d_{23}\right)$ to match the responses with the target ones derived from the coupling matrix. The dimensions associated with resonator 1 obtained in step 2 should be slightly adjusted to account for the influence of resonator 2 . This can be done with optimizations and has a fast convergence due to the final result being close to the optimum [1].

4. Progress through the filter structure by adding only one resonator into the simulated structure at each time, as illustrated in Figure 2. Optimize the dimensions of the subsequent resonator toward the desired S-parameter responses calculated from coupling matrix. A slight readjustment of the dimensions of the preceding resonators may be required to factor in the influence from the new added resonator. Normally, this small adjustment in dimensions is only required for adjacent resonators. For instance, at the last step [see Fig. 2 (d)], the dimensions of resonator 1 will remain the same as the ones obtained in step 3 , since resonator 4 has a negligible impact on resonator 1 .

\section{V.1 First Step:}

The first step in the design process takes exclusively into account only resonator 1 together with its two adjacent irises and resonator 2 is replaced with a Port 2 . The schematic of the circuit in the first step is illustrated in Figure 3(a). According to (10), the internal coupling $M_{1,2}$ is turned into its equivalent external coupling $M_{1, p 2}$ as

$$
M_{1, p 2}=\sqrt{\frac{n \pi}{2} F B W}\left(\frac{\lambda_{g}}{\lambda}\right) M_{1,2}=0.00642
$$

So the general $\mathrm{N}+2$ coupling matrix in step 1 is:

$$
\left[M_{1}\right]_{\mathrm{n}+2}=\left[\begin{array}{ccc}
0 & -0.0282 & 0 \\
-0.0282 & 0 & 0.00642 \\
0 & 0.00642 & 0
\end{array}\right]
$$

As given in Figure 3 (a), there are three physical dimensions (the width of the coupling irises $d_{01}$ and $d_{12}$, and the length of the cavity $l_{1}$ ) to be tuned. The aim of the tuning is to make the simulation results satisfy its objective counterpart obtained from the coupling matrix. The final results of First Step are given in Figure 3(b). The objective $S$-parameters in first step is derived from the coupling matrix in (11).

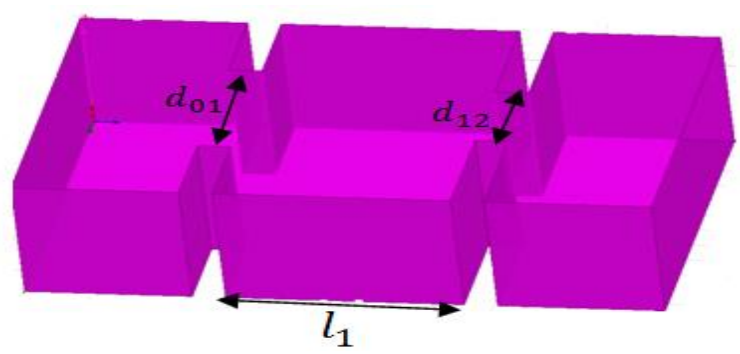

(a)

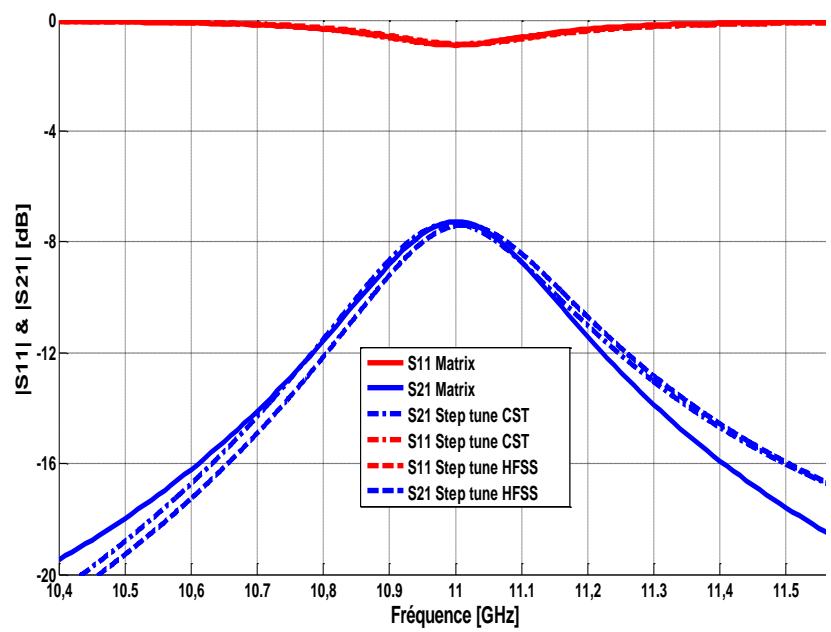

(b)

Fig.3. (a) The simulated physical structure in the first step. $d_{01}, d_{12}, l_{1}$ are the dimensions to tune. (b) Response comparison 


\section{V.2 Second Step:}

After completing the first step, another resonator is added successively and the focusing work moves to step two. The schematic of Step Two is given in Figure 2 (b). The equivalent coupling matrix of the schematic of Step Two Figure 2 (b) is

$$
M=\left[\begin{array}{cccc}
0 & -0.02822 & 0 & 0 \\
-0.2822 & 0 & 0.02491 & 0 \\
0 & 0.02491 & 0 & -0.00492 \\
0 & 0 & -0.00492 & 0
\end{array}\right]
$$

As given in Figure 4. (a), there are two new physical dimensions (width of the iris $d_{23}$ and the length of the cavity $l_{2}$ ) to be tuned. While the values of the old dimensions $\left(d_{01}, d_{12}, l_{1}\right)$ are slightly refined due to the loading effect of the new resonator. The comparison between the theoretical response (coupling matrix) and the EM simulation is shown in Figure 4 (b).

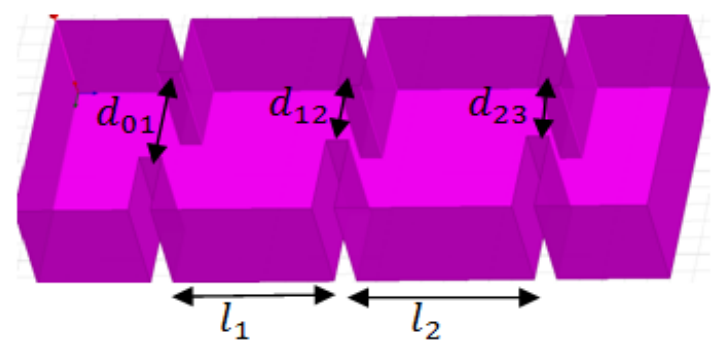

(a)

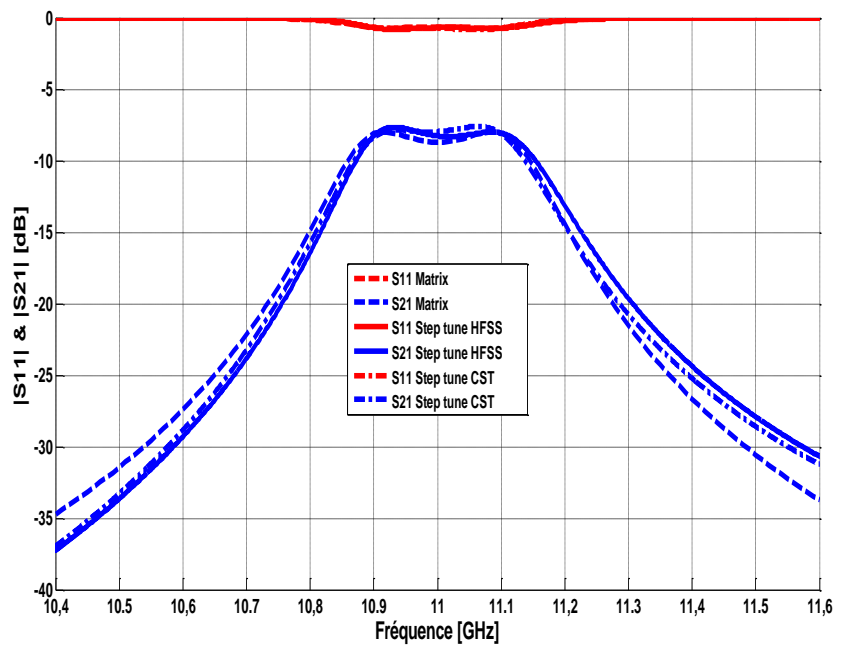

(b)

Fig. 4. (a) The simulated physical structure in the first step. $d_{23}, l_{2}$ are the dimensions to tune. (b) Response comparison between the tuned results and its objective ones

\section{V.3 Step Three:}

The third resonator is added in this step (the resonator 3 and characterized by the width of the iris $d_{34}$ and the length of the cavity $\left.1_{3}\right)$. While the values of the old dimensions are $\left(d_{01}, d_{12}, d_{23}, l_{1}, l_{2}\right)$. The rectangular waveguide shape in step (three) and given in Figure 4. Following the development, as shown in Figure 5 (b), the HFSS simulation reaction (in solid lines) approximates its response from the coupling matrix (in dashed lines). The equivalent coupling matrix of the scheme of step three is:

$$
M=\left[\begin{array}{ccccc}
0 & -0.0282 & 0 & 0 & 0 \\
-0.0282 & 0 & 0.0249 & 0 & 0 \\
0 & 0.0249 & 0 & -0.0191 & 0 \\
0 & 0 & -0.0191 & 0 & 0.00642 \\
0 & 0 & 0 & 0.00642 & 0
\end{array}\right]
$$

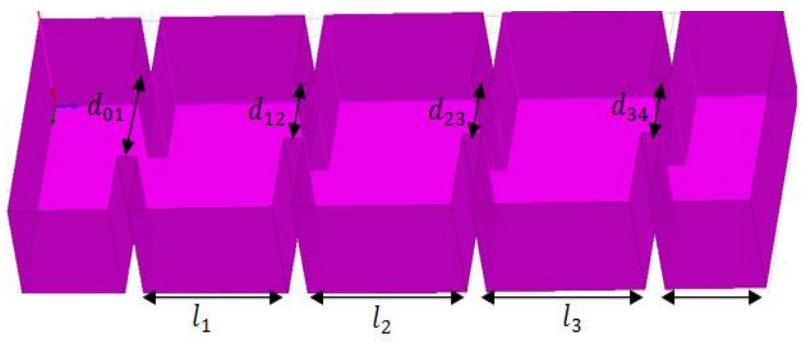

(a)

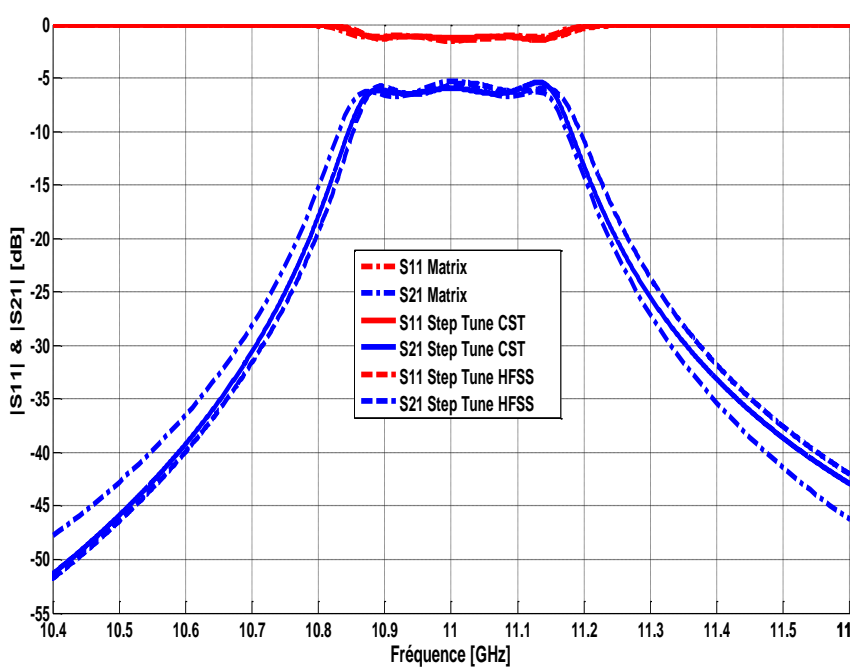

(b)

Fig. 5. (a) simulated physical structure in Step three. $d_{3,4}, l_{3}$ are the dimensions to tune. (b) Response comparison between the tuned results and its objective ones

V.4 Step Four:

Another resonator is added in this step (resonator 4 and characterized by the width of the iris $d_{45}$ and the length of the cavity $\left.l_{4}\right)$. The representation of the last step is given in Figure 6 (a).

$$
\begin{gathered}
M=\left[\begin{array}{cccccc}
0 & -0.0282 & 0 & 0 & 0 & 0 \\
-0.0282 & 0 & 0.0249 & 0 & 0 & 0 \\
0 & 0.0249 & 0 & -0.0191 & 0 & 0 \\
0 & 0 & -0.0191 & 0 & 0.0249 & 0 \\
0 & 0 & 0 & 0.0249 & 0 & -0.0282 \\
0 & 0 & 0 & 0 & -0.0282 & 0
\end{array}\right] \\
\mathrm{Q}_{\mathrm{e} 1}=\mathrm{Q}_{\mathrm{e} 2}=\mathrm{Q}_{\mathrm{e}}=34.21
\end{gathered}
$$




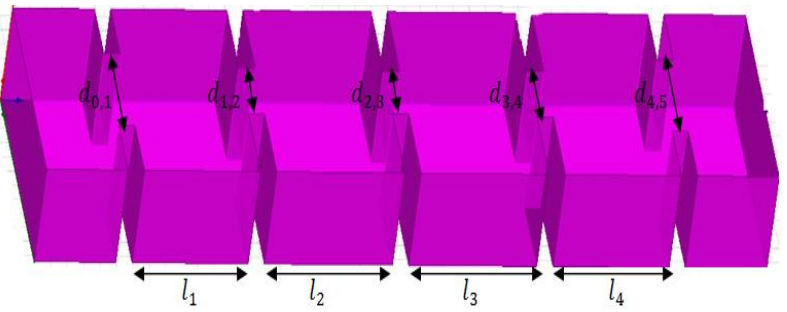

(a)

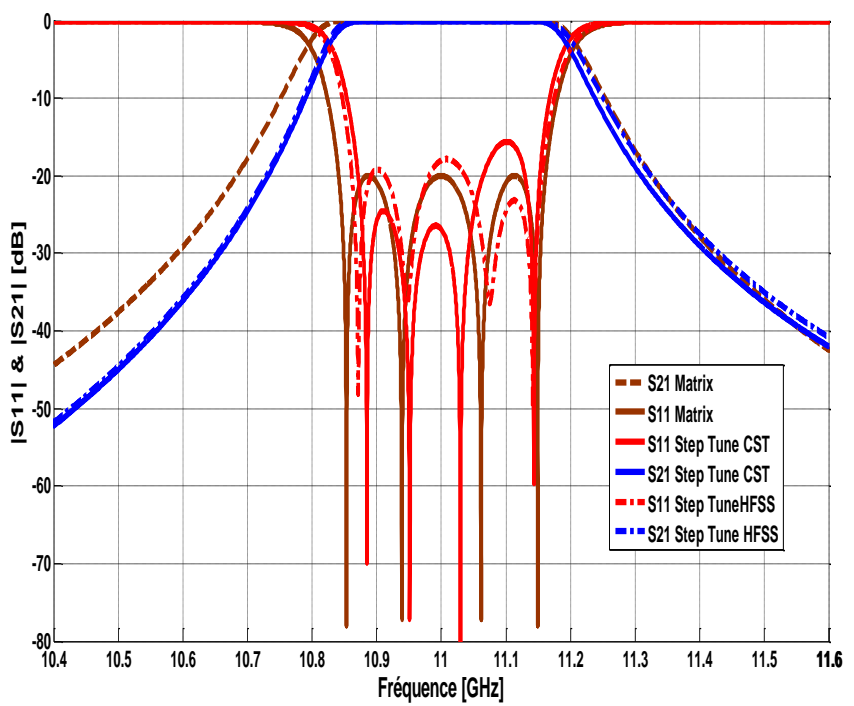

(b)

Fig.6 . (a) simulated physical structure in Step four. $d_{4,5}, l_{4}$ are the dimensions to tune. (b) Response comparison between the tuned results and its objective ones.

At each sub-step, initially only one resonator's dimensions (three or less parameters) are tuned in the simulations. Therefore, a desired set of dimensions, whose corresponding responses match the objective ones, can be obtained within a short time. The dimensions from the previous stages may also be slightly altered to tune the responses towards the desired ones, as shown in Tables I and II $[9,10]$.

TABLE I

BANDPASS FILTER IRIS DIMENSIONS AT EACH STEP.

\begin{tabular}{|l|c|l|l|l|l|}
\hline \multirow{2}{*}{ Step } & \multicolumn{6}{|c|}{ Dimension of iris (mm) } \\
\cline { 2 - 6 } & $d_{0,1}$ & $d_{1,2}$ & $d_{2,3}$ & \multicolumn{1}{|l}{$d_{3,4}$} & \multicolumn{1}{|c|}{$d_{4,5}$} \\
\hline 1 & 10.2 & 6.51 & - & - & - \\
2 & 10.22 & 6.51 & 6.02 & - & - \\
3 & 10.21 & 6.50 & 6.05 & 6.51 & - \\
4 & 10.21 & 6.51 & 6.02 & 6.51 & 10.21 \\
\hline
\end{tabular}

The final dimensions of the bandpass filter are shown in Tables I and II (Step 4) and their corresponding simulation results can be found in Figure 6 b. It can be observed that, without any global optimization, the final acquired dimensions have extremely close responses with the theory ones from coupling matrix.

Using the HFSS and CST softwares, the distribution of the electric field along the structure is given in Figure 7a.b.
TABLE II BANDPASS FILTER RESONATORS LENGTH AT EACH.

\begin{tabular}{|l|l|l|l|l|}
\hline \multirow{2}{*}{ Step } & \multicolumn{4}{|l|}{ Length of resonators $(\mathbf{m m})$} \\
\cline { 2 - 5 } & \multicolumn{1}{|c|}{$l_{1}$} & \multicolumn{1}{|c|}{$l_{2}$} & \multicolumn{1}{c|}{$l_{3}$} & \multicolumn{1}{c|}{$l_{4}$} \\
\hline 1 & 14.249 & - & - & - \\
2 & 14.249 & 15.72 & - & - \\
3 & 14.24 & 15.71 & 15.7 & - \\
4 & 14.24 & 15.71 & 15.71 & 14.24 \\
\hline
\end{tabular}

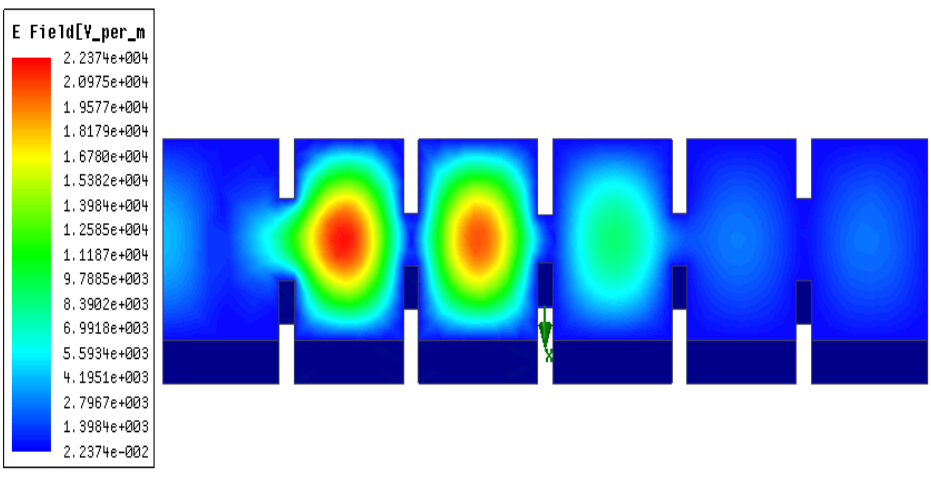

Figure 7.a: Electrical fields simulated by HFSS at frequency $11 \mathrm{GHz}$.

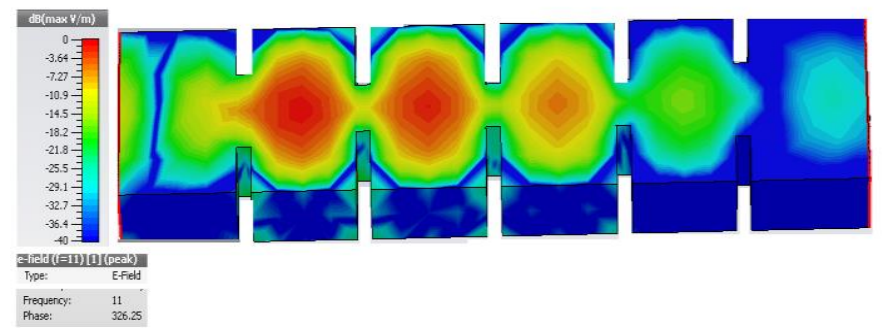

Figure 7.b: Electrical fields simulated by CST at frequency $11 \mathrm{GHz}$.

\section{CONCLUSION}

This article illustrates how to design the four-pole Chebyshev inductive iris coupled resonator based rectangular waveguide circuit in the traditional way. The filter structure is constructed step by step by adding one resonator to the simulated structure at a time. Equations have been derived and provided in this article to plot the middle stage responses from coupling matrix With a large number of variables, such tuning work consumes a lot of time and the convergence of the final result is not guaranteed. This section ends with physical realization of dirctcoupled waveguide filters based on electromagnetic (EM) simulations using step tune method.

\section{REFERENCES}

[1] Shang, X, Xia, W \& Lancaster, MJ 2014, 'The design of waveguide filters based on cross-coupled resonators' Microwave and Optical Technology Letters, vol 56, no. 1, pp. 3-8., 10.1002/mop.27989

[2] Cameron, C.M. Kudsia, and R.R. Mansour, Microwave filters for commmunication systems, Wiley, Hoboken, NJ, 2007.

[3] W. Xia, X. Shang, and M. J. Lancaster., "Responses comparisons for coupled-resonator based diplexers," in Passive RF and Microwave Components, 3rd Annual Seminar on, 2012, pp. 67-75

[4] Xiaobang Shang, Wenlin Xia, and Michael J Lancaster. 'The design of waveguide filters based on cross-coupled resonators. Microwave and Optical Technology Letters, 56(1):3-8,2014

[5] X. Shang, Y. Wang, G.L. Nicholson, and M.J. Lancaster, Design of multiple-passband filters using coupling matrix optimisation, IET Microwave Antenna Propag 6 (2012), 24-30. 
[6] G. Macchiarella, "An efficient technique for the synthesis of an equiripple low-pass prototype filter with asymmetric frequency response and arbitrary transfer function zeros," in 24th European Microwave Conf., Bologna, Italy, Sept. 1995, pp. 722-724.

[7] Xiaobang Shang, Wenlin Xia, and Michael J Lancaster. The design of waveguide filters based on cross-coupled resonators. Microwave and Optical Technology Letters, 56(1):3-8, 2014
[8] F.M. Vanin and D. Schmitt, R. Levy, Dimensional synthesis for wideband waveguide filters, In: IEEE MTT-S International Microwave Symposium, Fort Worth, TX, June 2004, 463-466

[9] User's guide - High Frequency Structure Simulator (HFSS), v13.0, Ansoft. [10]CST Microwave Studio Germany, CST GmbH, 2014 